\title{
Composição florística da floresta estacional decídua montana de Serra das Almas, CE, Brasil ${ }^{1}$
}

\author{
Jacira Rabelo Lima ${ }^{2}$, Everardo Valadares de Sá Barretto Sampaio ${ }^{3}$, Maria Jesus Nogueira Rodal ${ }^{4}$ e \\ Francisca Soares Araújo ${ }^{5,6}$
}

Recebido em 5/11/2007. Aceito em 10/11/2008

\begin{abstract}
RESUMO - (Composição florística da floresta estacional decídua montana de Serra das Almas, CE, Brasil). No domínio semi-árido brasileiro, a flora das bacias sedimentares ainda é pouco conhecida, mas os levantamentos já existentes indicam que há grande heterogeneidade florística e fisionômica. Mesmo áreas geográficas próximas podem apresentar dissimilaridade florística. Visando testar esta hipótese, a composição florística e o espectro biológico da floresta estacional decídua de Serra das Almas, estado do Ceará, foram analisados e comparados com os de 14 áreas sedimentares no Nordeste. Foram encontradas 104 espécies e 39 famílias em 1 ha analisado. Fabaceae, Euphorbiaceae, Erythroxylaceae e Myrtaceae foram as famílias com maior riqueza e fanerófitos a forma de vida predominante (87\%). Arbóreas e arbustivas representaram $72 \%$ das espécies, trepadeiras 15\%, subarbustos $6 \%$ e herbáceas $7 \%$. A análise de agrupamento da composição dos fanerófitos, usando o índice de Jaccard e as médias de grupo a posteriori, indicou a formação de grupos florísticos entre áreas geográficas mais próximas. Porém, não foi significativo pelo teste de Mantel, o que demonstra a ocorrência de alta heterogeneidade florística mesmo entre áreas geográficas próximas. A flora da floresta da Serra das Almas apresentou maior semelhança com as formações encontradas no planalto da Ibiapaba.
\end{abstract}

Palavras-chave: flora, floresta tropical, formas de vida, semi-árido

ABSTRACT - (Flora of seasonal deciduous montane forest at Serra das Almas, Ceará State, Brazil). In the Brazilian semi-arid region, the flora in the sedimentary basins has been poorly studied, but the few surveys already done indicate great floristic and physiognomic heterogeneity. Even neighboring sites may be floristically different. To test this hypothesis, the floristic composition and the biological spectrum of the seasonal deciduous forest at Serra das Almas, Ceará, was analyzed and compared to those of 14 other northeastern Brazil sedimentary areas. A total of 104 species belonging to 40 families were found at the 1 ha site. Fabaceae, Euphorbiaceae, Erythroxylaceae and Myrtaceae were the families with the most species and phanerophytes the main life form, comprising $87 \%$ of the species. About $72 \%$ of the species were trees and shrubs, $15 \%$ were lianas, $6 \%$ understory shrubs and $7 \%$ herbs. A cluster analysis of the woody flora of the 14 areas, using the Jaccard index and a posteriori group averages, indicated the formation of groups as a function of geographical proximity of the areas. However, this effect was not significant, according to the Mantel test, demonstrating floristic heterogeneity even in neighboring areas. The flora of the forest at Serra das Almas was most similar to that of the vegetation found atop the Ibiapaba plateau.

Key words: dry forest, flora, plant life-form, semi-arid

\section{Introdução}

A vegetação é o componente dominante e mais acessível das comunidades ecológicas terrestres (Shimwell 1971). Por isso, existem diversas propostas de classificação com base em diferentes critérios e escalas espaciais (Whitaker 1975). De acordo com Kent \& Coker (1994), é necessário distinguir entre sistemas de classificação da vegetação que empregam atributos de composição de espécies, formas de vida, características foliares e mecanismos de dispersão daqueles sistemas de classificação de ecossistemas, que empregam dados das principais características ambientais, tais como solos, hidrologia, relevo e cobertura vegetal. Sistemas que têm por objetivo classificar a vegetação, geralmente florísticos ou fisionômicos, propõem fornecer a ordenação sistemática dos padrões da vegetação e relacionar esses padrões aos processos ecológicos. Sistemas fisionômicos são divisivos e, em diferentes escalas, têm demonstrado que há relação entre os tipos fisionômicos e as características climáticas, especialmente, precipitação e temperatura. Sistemas florísticos, basicamente aglomerativos, partem das espécies dominantes e freqüentes e revelam padrões mais detalhados que os fisionômicos.

A possibilidade de realizar ajuste entre sistemas fisionômicos e florísticos é desejável, pois permite o mapeamento (fisionomia) de caracteres vinculados a determinada área onde há informações sobre aspectos florísticos. Como exemplo de tentativa do ajuste de classificação fisionômica e florística tem-se a proposta de Veloso et al. (1991) para a vegetação brasileira. Nessa, são reconhecidas quatro regiões florísticas, subdivididas com base em critérios fisionômicos e ecológicos, principalmente, no espectro biológico. Na região da savana-estépica, que ocupa grosseiramente os mesmos limites do semi-árido nordestino, predomina a caatinga sensu stricto. Trata-se da tipologia vegetal (área com fisionomia e flora distintas das áreas adjacentes), de maior extensão no semi-árido, caracterizada pela presença de fanerófitos de pequeno porte, caméfitos e terófitos (Brasil 1981), que ocorre especialmente nas terras baixas entre serras e planaltos, a chamada

\footnotetext{
1 Parte da Dissertação da primeira Autora

2 Jardim Botânico do Rio de Janeiro, Escola Nacional de Botânica Tropical, Programa de Pós-graduação em Botânica, Rio de Janeiro, RJ, Brasil

3 Universidade Federal de Pernambuco, Departamento de Energia Nuclear, Recife, PE, Brasil

4 Universidade Federal Rural de Pernambuco, Departamento de Biologia, Recife, PE, Brasil

5 Universidade Federal do Ceará, Campus do Pici, Centro de Ciências, Departamento de Biologia, Fortaleza, CE, Brasil

6 Autor para correspondência: tchesca@ufc.br
} 
depressão sertaneja, a qual ocupa $368.216 \mathrm{~km}^{2}$ (para revisão ver Souza et al. 1994).

Sob o mesmo domínio climático, a dissimilaridade florística entre diferentes tipologias fisionômicas é maior entre unidades geomorfológicas distintas (por exemplo: embasamento cristalino versus bacias sedimentares) do que entre floras de tipologias da mesma unidade geomorfológica (Araújo et al. 2005a; b). Dentro da mesma unidade geomorfológica, o efeito da distância geográfica na distribuição das espécies predomina sobre o clima e, portanto, áreas mais próximas tendem a apresentar maior semelhança florística, independente das condições climáticas (Moura \& Sampaio 2001; Barbosa et al. 2004).

No domínio da savana-estépica, estudos em escala mais detalhada demonstram que a flora da depressão sertaneja é distinta da que ocorre nas serras cristalinas (ver revisão de Sampaio 1996; Gadelha Neto \& Barbosa 2000; Pereira et al. 2002; Alcoforado Filho et al. 2003; Ferraz et al. 2003; Nascimento et al. 2003; Araújo et al. 2005a), onde há registro de formações florestais com diferentes níveis de caducifólia e que tiveram levantamento florístico iniciado em estudos pontuais sobre áreas situadas em habitats secos e interioranos (Ferraz et al. 1998; Moura \& Sampaio 2001; Ferraz et al. 2003; Nascimento \& Rodal 2008) e em habitats úmidos e subúmidos próximos da costa atlântica (Tavares et al. 2000; Barbosa et al. 2004; Rodal et al. 2005; Ferraz \& Rodal 2006; Ferraz \& Rodal 2008). A flora da depressão sertaneja também difere da encontrada nas bacias sedimentares do semi-árido (Araújo et al. 2005a), onde há registro de formações florestais estacionais (Rodal \& Nascimento 2002) e não florestais, como o carrasco vegetação arbustiva caducifólia não-espinhosa (Araújo et al. 1998a; b; Araújo et al. 1999), o cerrado (Costa et al. 2004) e áreas ecotonais de vegetação decídua entre a caatinga e o cerrado (Oliveira et al. 1997). Vale destacar que no domínio semi-árido brasileiro, a flora das bacias sedimentares ainda é pouco conhecida, mas os levantamentos já existentes indicam que há grande heterogeneidade florística e fisionômica. Diante dos indícios, ainda preliminares, da grande heterogeneidade fisionômica-florística nas bacias sedimentares, as áreas geograficamente próximas, apresentam baixa similaridade florística.

Portanto, o objetivo deste trabalho foi conhecer a composição e o espectro biológico da flora de um hectare da floresta estacional decídua montana (floresta seca) sobre a vertente oriental do sul do planalto da Ibiapaba e analisar a semelhança com a flora de diferentes tipologias fisionômicas localizadas sobre áreas sedimentares do domínio semi-árido brasileiro (Bacia Sedimentar do Meio Norte, Tucano-Jatobá, Chapada do Araripe e Apodi). Com isso pretende-se responder às seguintes questões: quais espécies compõem a floresta estacional decídua montana do planalto da Ibiapaba? A flora da área estudada apresenta maior similaridade com formações vegetacionais geograficamente mais próximas?

\section{Material e métodos}

Localização e caracterização ambiental da área de estudo - A área de estudo foi a Reserva Natural Serra das Almas (RNSA), situada na parte centro-sul do planalto da Ibiapaba, que é o bordo oriental da bacia sedimentar do Meio Norte (Lins 1978), na divisa do Ceará e do Piauí, no município de Crateús, Ceará. A reserva foi criada em 1998 (portaria $\mathrm{n}^{\circ}$ 51/00 IBAMA) e abrange uma área de 5646 ha. Na RNSA ocorrem três formações vegetacionais: caatinga, carrasco e floresta estacional decídua. A floresta estacional ocupa uma área de aproximadamente 2.790 ha, dos quais ca. 1.400 ha encontram se em um bom estado de conservação. Foi delimitada uma área de 1 ha na floresta, no interior de um fragmento conservado de 2,8 ha a uma altitude de cerca de $650 \mathrm{~m}$, entre as coordenadas $5^{\circ} 8^{\prime} 29^{\prime \prime} \mathrm{S}$ e $40^{\circ} 54^{\prime} 51^{\prime \prime} \mathrm{W} ; 5^{\circ} 8^{\prime} 30^{\prime \prime} \mathrm{S}$ e $40^{\circ} 54^{\prime} 45^{\prime \prime} \mathrm{W}$; $5^{\circ} 8^{\prime} 36^{\prime \prime} \mathrm{S}$ e $40^{\circ} 54^{\prime} 46^{\prime \prime} \mathrm{W}$ e $5^{\circ} 8^{\prime} 35^{\prime \prime} \mathrm{S}$ e $40^{\circ} 54^{\prime} 50^{\prime \prime} \mathrm{W}$.

A vegetação pela classificação de Veloso et al. (1991) é de floresta estacional decídua, caracterizada por estar submetida a duas estações climáticas: uma chuvosa e outra com um longo período seco, quando mais de $50 \%$ dos indivíduos perdem suas folhas. Na floresta estudada foram observados dois estratos: um dominado por indivíduos de até $12 \mathrm{~m}$ de altura e outro, mais baixo, com indivíduos de no máximo $8 \mathrm{~m}$, formando uma copa contínua. Alguns indivíduos de Brosimum gaudichaudii, Piptadenia moniliformis e Swartzia flaemingii alcançam até $18 \mathrm{~m}$, sendo considerados como emergentes na comunidade.

A precipitação média anual no posto pluviométrico instalado na sede da reserva, distante aproximadamente $200 \mathrm{~m}$ do hectare estudado, nos anos de 2000 a 2004, foi de $1044 \mathrm{~mm}$, sendo janeiro a abril os meses mais chuvosos, correspondendo a mais de $80 \%$ da precipitação anual. Em geral, houve uma maior concentração das chuvas em um único mês e ausência quase completa no período de julho a dezembro. A temperatura média mensal, obtida na mini-estação meteorológica localizada na Reserva, para o ano de 2004 , foi de $24,8{ }^{\circ} \mathrm{C}$.

Composição florística - $\mathrm{Na}$ área do hectare delimitado, foi realizado o censo de todos os indivíduos lenhosos que tinham caule com mais de $3 \mathrm{~cm}$ de diâmetro. Destes indivíduos e de todas as outras angiospermas arbustivas, trepadeiras e herbáceas, foram feitas coletas de ramos em fase reprodutiva, para preparação de, no mínimo, cinco duplicatas. Também foram coletados ramos vegetativos das espécies nas quais não foi possível encontrar ramos em estado reprodutivo. Para cada espécime foi registrado hábito com base em Whittaker (1975) e classificação da forma de vida da espécie, segundo Raunkiaer (1934). Para facilitar a coleta, o hectare estudado foi subdividido em 100 parcelas de $10 \times 10 \mathrm{~m}^{2}$. A partir da classificação das espécies por forma de vida, foi calculado o espectro biológico da área e comparado com o levantamento em uma área de carrasco (Serra das Almas, Ceará, Araújo et al. 2005b), através do teste Qui-quadrado $\left(\mathrm{X}^{2}\right)$.

O material testemunho foi incorporado ao herbário EAC (Prisco Bezerra), da Universidade Federal do Ceará. A determinação botânica foi realizada com auxílio de chaves analíticas (Freire 1983; Barroso et al. $1978 ; 1984 ; 1986)$ e por comparação com material existente nos Herbários EAC e PEUFR, ou, quando necessário, através de envio a especialistas. O sistema de classificação adotado foi o APG II (2003). Os nomes das espécies foram atualizados quanto à sinonímia, consultando-se o sítio do Missouri Botanical Garden (http:// www.mobot.org, acesso em 14/10/2007) e os nomes e/ou abreviaturas dos autores das espécies foram grafados de acordo com Brummitt \& Powell (1992).

Similaridade florística - Para analisar a semelhança florística entre a vegetação estudada e outras formações localizadas em áreas sedimentares no Nordeste semi-árido (Fig. 1), independente da fisionomia, foi elaborada uma matriz binária (presença/ausência) dos táxons arbóreos e arbustivos identificados no nível de espécie, presentes em 14 áreas de 17 levantamentos (L), tanto com formações de fisionomia não-florestal como florestal. As áreas sedimentares com formações não-florestais foram Oliveira et al. (1997): L1; Araújo et al. (1998a): L2 a L4; Araújo et al. (1999): L5; Rodal et al. (1999): L6; Figueirêdo et al. (2000): L7; Lemos \& Rodal (2002): L8; Andrade et al. (2004): L9; Farias \& Castro (2004): L10 e L11; Rocha et al. (2004): L12; Gomes et al. (2006): L13; Costa et al. (2004): L14; Maracajá et al. (2003): L15; e M.R.A. Mendes (dados não publicados): 


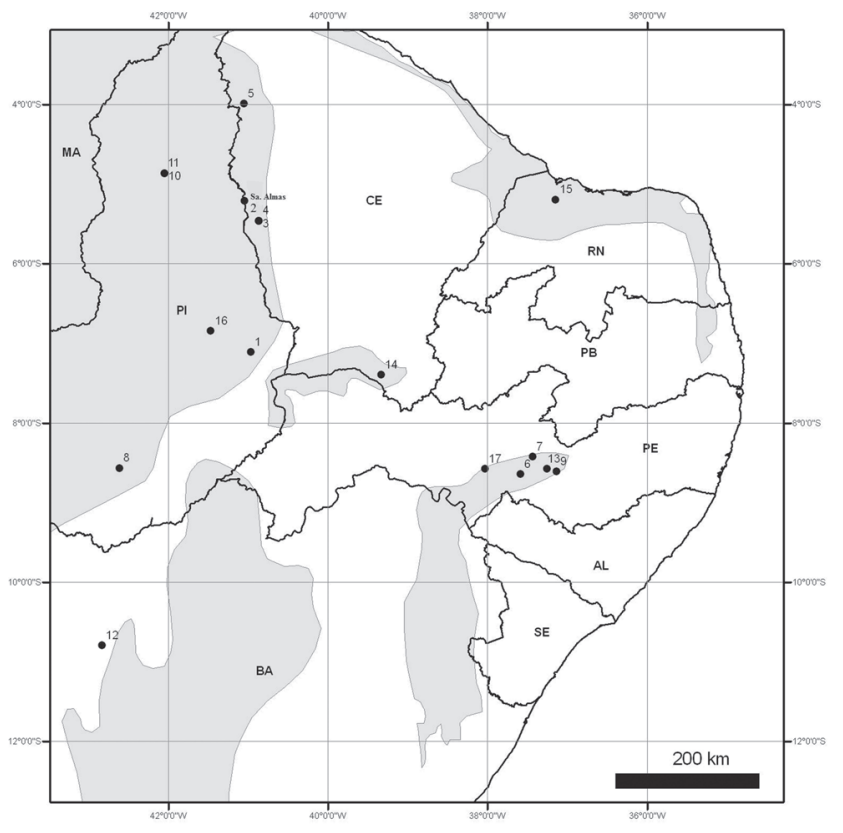

Figura 1. Mapa do Nordeste brasileiro mostrando a localização da Floresta Estacional Decídua Montana da Serra das Almas, Ceará (Sa. Almas) e os levantamentos em área sedimentares (cor cinza) usados na análise de similaridade. Os levantamentos foram os seguintes: 1: Oliveira et al. (1997); 2 a 4: Araújo et al. (1998a); 5: Araújo et al. (1999); 6: Rodal et al. (1999); 7: Figueirêdo et al. (2000); 8: Lemos \& Rodal (2002); 9: Andrade et al. (2004); 10 e 11: Farias \& Castro (2004); 12: Rocha et al. (2004); 13: Gomes et al. (2006); 14: Costa et al. (2004); 15: Maracajá et al. (2003); 16: M.R.A. Mendes (dados não publicados) e 17: Rodal \& Nascimento (2002).
L16. A área com formação florestal foi Rodal \& Nascimento (2002): L17.

A partir da matriz binária foi calculada a matriz quadrada e simétrica de similaridade de Jaccard (Krebs 1989). A construção do dendrograma resultante da análise de aglomerado foi realizada através da média de grupo (Krebs 1989), usando o programa FITOPAC (Shepherd 2006). Para testar se os índices de similaridade e, conseqüentemente, se o agrupamento entre as áreas não é explicado pelo acaso, foram realizadas 1000 replicações a partir do método de simulação Monte Carlo, através do RandMat 1.0 for Windows (http://eco.ib.usp.br/labmar), adotando se o nível de $1 \%$ de probabilidade. Para avaliar a relação entre a similaridade florística e a distância geográfica, foi realizado o teste de Mantel, usando o programa Mantel 2.0 (Liedloff 1999).

\section{Resultados}

Composição florística - A flora da área estudada, incluindo ervas, subarbustos, arbustos, árvores e trepadeiras, foi representada por 104 espécies, pertencentes a 76 gêneros e 39 famílias (Tab. 1). As famílias com maior riqueza de espécies foram Fabaceae (20 espécies), Euphorbiaceae (11 espécies), Erythroxylaceae e Myrtaceae (seis espécies cada), Apocynaceae e Bignoniaceae (cinco espécies cada) e Rubiaceae (quatro espécies). Das 39 famílias, 20 (50\%) foram representadas por uma única espécie e nove $(23 \%)$ por duas espécies. Os gêneros com maior número de espécies foram Erythroxylum (seis espécies), Croton e Eugenia (cinco espécies cada) e Arrabidea e Aspidosperma (três espécies cada).

Fanerófitos foram a forma de vida predominante, responsáveis por $87 \%$ das espécies, seguidos por caméfitos

Tabela 1. Lista de famílias e espécies que ocorrem em um hectare de floresta estacional decídua montana da Reserva Natural Serra das Almas, município de Crateús, Ceará, com seus respectivos hábitos, segundo a classificação de Whittaker (1975), formas de vida, segundo a classificação de Raunkiaer (1934), nome e número de coletor e grupo de distribuição. Hábito: arv - árvore; arb - arbusto; arb esc - arbusto escandente; erv - erva; sub - subarbusto e trp - trepadeira. Formas de vida (FV): Ch - caméfito; H - hemicriptófito; $\mathrm{Cg}$ - criptófito (geófito); Ph - fanerófito e Th - terófito.

\begin{tabular}{|c|c|c|c|c|c|c|c|}
\hline Família/Espécie & Hábito & FV & Nome e $\mathrm{N}^{\circ}$ Coletor & Família/Espécie & Hábito & FV & Nome e $\mathrm{N}^{\circ}$ Coletor \\
\hline ACANTHACEAE & & & & BIXACEAE & & & \\
\hline Justicia fragilis Wall. ex Clarke & Sub & $\mathrm{Ch}$ & J.R.Lima, 14 & Cochlospermum vitifolium & Arv & $\mathrm{Ph}$ & S.F.Vasconcelos, 4 \\
\hline J. strobilacea (Nees) Lindau & Arb & $\mathrm{Ph}$ & J.R.Lima, 15 & (Willd.) Spreng. & & & \\
\hline ACHARIACEAE & & & & BORAGINACEAE & & & \\
\hline Lindackeria ovata (Benth.) Gilg & Arv & $\mathrm{Ph}$ & J.R.Lima, 76 & Cordia rufescens A. DC. & Arb & $\mathrm{Ph}$ & J.R.Lima, 24 \\
\hline ANNONACEAE & & & & BROMELIACEAE & & & \\
\hline Ephedranthus pisocarpus R. E. Fr. & Arv & $\mathrm{Ph}$ & J.R.Lima, 16 & Bromelia auriculata L.B. Sm. & Erv & $\mathrm{H} \mathrm{I}$ & L.W. Lima-Verde, 1222 \\
\hline $\begin{array}{l}\text { Rollinia leptopetala } \text { R. E. Fr. } \\
\text { APOCYNACEAE }\end{array}$ & Arb & $\mathrm{Ph}$ & J.R.Lima, 78 & $\begin{array}{l}\text { B. plumieri (E. Morren) L.B. Sm. } \\
\text { BURSERACEAE }\end{array}$ & Erv & $\mathrm{H}$ & F.S.Araújo, 353 \\
\hline Aspidosperma discolor A. DC. & Arv & $\mathrm{Ph}$ & J.R.Lima, 18 & Commiphora leptophloeos (Mart.) & Arv & $\mathrm{Ph}$ & J.R.Lima, 48 \\
\hline A. multiflorum A. DC. & Arv & $\mathrm{Ph}$ & J.R.Lima, 19 & J.B. Gillett & & & \\
\hline A. subincanum Mart. & Arv & $\mathrm{Ph}$ & J.R.Lima, 17 & CACTACEAE & & & \\
\hline Secondantia cf. foliosa A. DC. & $\operatorname{Trp}$ & $\mathrm{Ph}$ & J.R.Lima, 89 & Cereus jamacaru DC. & Arb & $\mathrm{Ph}$ & F.S.Araújo, 382 \\
\hline Tassadia burchelii E. Fourn. & Trp & $\mathrm{Ph}$ & J.R.Lima, 13 & CELASTRACEAE & & & \\
\hline ARACEAE & & & & Maytenus sp. & Arv & $\mathrm{Ph}$ & J.R.Lima, 100 \\
\hline Scaphispatha hastifolia Hook. & Erv & $\mathrm{Cg}$ & J.R.Lima, 105 & COMBRETACEAE & & & \\
\hline ASTERACEAE & & & & Buchenavia tetraphylla (Aubl.) & Arv & $\mathrm{Ph}$ & M.S.Sobrinho, 292 \\
\hline Wedelia villosa Gardner & Sub & $\mathrm{Ch}$ & J.R.Lima, 85 & R.A. Howard & & & \\
\hline BIGNONIACEAE & & & & Combretum leprosum Mart. & Arb Esc & $\mathrm{Ph}$ & J.R.Lima, 74 \\
\hline $\begin{array}{l}\text { Arrabidaea chica (Humb. \& } \\
\text { Bonpl.) B. Verl. }\end{array}$ & $\operatorname{Trp}$ & $\mathrm{Ph}$ & J.R.Lima, 21 & $\begin{array}{l}\text { Thiloa glaucocarpa (Mart.) Eichler } \\
\text { CONVOLVULACEAE }\end{array}$ & Arv & $\mathrm{Ph}$ & L.W.Lima-Verde, 1050 \\
\hline A. dispar Bureau ex K. Schum. & Trp & $\mathrm{Ph}$ & J.R.Lima, 20 & Evolvulus macroblepharis Mart. & Sub & $\mathrm{Ch}$ & J.R.Lima, 83 \\
\hline Arrabidaea sp. & $\operatorname{Trp}$ & $\mathrm{Ph}$ & J.R.Lima, 107 & Ipomoea brasiliana Meisn. & Trp & $\mathrm{Ph}$ & J.R.Lima, 25 \\
\hline Jacaranda jasminoides (Thunb.) & Trp & $\mathrm{Ph}$ & J.R.Lima, 22 & CYPERACEAE & & & \\
\hline Sandwith & & & & Cyperus aggregatus (Willd.) Endl. & Erv & $\mathrm{H}$ & J.R.Lima, 106 \\
\hline $\begin{array}{l}\text { Tabebuia cf. ochracea (Cham.) } \\
\text { Standl. }\end{array}$ & Arv & $\mathrm{Ph}$ & J.R.Lima, 23 & $\begin{array}{l}\text { ERYTHROXYLACEAE } \\
\text { Erythroxylum barbatum O.E. Schulz }\end{array}$ & Arb & $\mathrm{Ph}$ & J.R.Lima, 67 \\
\hline
\end{tabular}


Tabela 1 (continuação)

\begin{tabular}{|c|c|c|c|c|c|c|c|}
\hline Família/Espécie & Hábito & FV & Nome e $\mathrm{N}^{\circ}$ Coletor & Família/Espécie & Hábito & FV & Nome e $\mathrm{N}^{\circ}$ Coletor \\
\hline E. bezerrae Plowman & Arb & $\mathrm{Ph}$ & J.R.Lima, 68 & H. muscosa Mart. & Arb & $\mathrm{Ph}$ & J.R.Lima, 52 \\
\hline E. cf. vacciniifolium Mart. & Arb & $\mathrm{Ph}$ & J.R.Lima, 69 & MELIACEAE & & & \\
\hline E. laetevirens O.E. Schulz & Arb & $\mathrm{Ph}$ & J.R.Lima, 66 & Trichilia elegans A. Juss. & Arb & $\mathrm{Ph}$ & J.R.Lima, 31 \\
\hline Erythroxylum sp. & Arb & $\mathrm{Ph}$ & J.R.Lima, 87 & MORACEAE & & & \\
\hline E. stipulosum Plowman & Arb & $\mathrm{Ph}$ & J.R.Lima, 86 & Brosimum gaudichaudii Trécul & Arv & $\mathrm{Ph}$ & Probio, 306 \\
\hline EUPHORBIACEAE & & & & MYRTACEAE & & & \\
\hline Cnidoscolus vitifolius (Mill.) Pohl & Arb & $\mathrm{Ph}$ & F. S. Araújo, 1309 & Campomanesia aromatica & Arb & $\mathrm{Ph}$ & J.R.Lima, 61 \\
\hline Croton argyrophylloides Müll Arg. & Arb & $\mathrm{Ph}$ & F. S. Araújo, 1294 & (Aubl.) Griseb. & & & \\
\hline C. betaceus Baill. & Arb & $\mathrm{Ph}$ & J.R.Lima, 81 & Eugenia aff. dysenterica DC. & Arv & $\mathrm{Ph}$ & F.S.Araújo, 1291 \\
\hline C. blanchetianus Baill. & Arb & $\mathrm{Ph}$ & J.R.Lima, 82 & E. aff. uvalha Cambess. & Arb & $\mathrm{Ph}$ & J.R.Lima, 73 \\
\hline C. grewioides Baill. & Arb & $\mathrm{Ph}$ & J.R.Lima, 79 & E. flavescens DC. & Arv & $\mathrm{Ph}$ & J.R.Lima, 60 \\
\hline C. nepetifolius Baill. & Arb & $\mathrm{Ph}$ & J.R.Lima, 28 & E. piauhiensis O. Berg & Arv & $\mathrm{Ph}$ & J.R.Lima, 62 \\
\hline Euphorbia comosa Vell. & Arb & $\mathrm{Ph}$ & F.S.Araújo, 1286 & E. punicifolia (Kunth) DC. & Arv & $\mathrm{Ph}$ & J.R.Lima, 63 \\
\hline Gymnanthes sp.1 & Arb & $\mathrm{Ph}$ & J.R.Lima, 29 & NYCTAGINACEAE & & & \\
\hline Gymnanthes sp. 2 & Arv & $\mathrm{Ph}$ & J.R.Lima, 27 & Guapira graciliflora & Arv & $\mathrm{Ph}$ & J.R.Lima, 34 \\
\hline Manihot palmata Mull. Arg. & Arb Esc & $\mathrm{Ph}$ & J.R.Lima, 26 & (Schmidt) Lundell & & & \\
\hline Sapium aff. argutum (Müll. Arg.) & Arv & $\mathrm{Ph}$ & J.R.Lima, 75 & Ouratea cf. parvifolia Engl. & Arv & $\mathrm{Ph}$ & J.R.Lima, 53 \\
\hline Huber & & & & Ouratea sp. & Arv & $\mathrm{Ph}$ & J.R.Lima, 54 \\
\hline FABACEAE & & & & OLACACEAE & & & \\
\hline Acacia langsdorffii Benth & Arb & $\mathrm{Ph}$ & J.R.Lima, 40 & Ximenia americana $\mathrm{L}$. & Arv & $\mathrm{Ph}$ & J.R.Lima, 55 \\
\hline Amburana cearensis (Allemão) & Arv & $\mathrm{Ph}$ & J.R.Lima, 72 & OPILIACEAE & & & \\
\hline A.C. Sm. & & & & Agonandra brasiliensis Miers & Arv & $\mathrm{Ph}$ & J.R.Lima, 56 \\
\hline Bauhinia pulchella Benth. & Arv & $\mathrm{Ph}$ & J.R.Lima, 45 & POACEAE & & & \\
\hline Copaifera martii Hayne & Arv & $\mathrm{Ph}$ & J.R.Lima, 38 & Lasiacis sorghoidea (Desv. ex & Erv & Th & J.R.Lima, 92 \\
\hline Cranocarpus gracilis Afr. Fern. & Sub & $\mathrm{Ch}$ & J.R.Lima, 84 & Ham.) Hitchc. \& Chase & & & \\
\hline \& P. Bezerra & & & & Streptostachys asperifolia Desv. & Erv & $\mathrm{Th}$ & F.S.Araújo, 1307 \\
\hline Dalbergia cearensis Ducke & Arv & $\mathrm{Ph}$ & L.W.Lima-Verde, 1070 & & & & continua \\
\hline Dalbergia sp. & Arb Esc & $\mathrm{Ph}$ & J.R.Lima, 41 & POLYGALACEAE & & & \\
\hline Dioclea megacarpa Rolfe & Trp & $\mathrm{Ph}$ & J.R.Lima, 37 & Bredemeyera floribunda Willd. & Trp & $\mathrm{Ph}$ & J.R.Lima, 57 \\
\hline Hymenaea eriogyne Benth. & Arv & $\mathrm{Ph}$ & J.R.Lima, 42 & RHAMNACEAE & & & \\
\hline H. velutina Ducke & Arv & $\mathrm{Ph}$ & J.R.Lima, 43 & Colubrina cordifolia Reissek & Arb & $\mathrm{Ph}$ & M.S.Sobrinho, 248 \\
\hline Lonchocarpus araripensis Benth. & Arv & $\mathrm{Ph}$ & J.R.Lima, 49 & Gouania sp. & $\operatorname{Trp}$ & $\mathrm{Ph}$ & J.R.Lima, 58 \\
\hline Luetzelburgia auriculata (Allemão) & Arv & $\mathrm{Ph}$ & J.R.Lima, 71 & RUBIACEAE & & & \\
\hline Ducke & & & & Alibertia myrciifolia Spruce & Arv & $\mathrm{Ph}$ & J.R.Lima, 102 \\
\hline Machaerium acutifolium Vogel & Arv & $\mathrm{Ph}$ & J.R.Lima, 80 & ex K. Schum. & & & \\
\hline Mimosa sp. & Arb & $\mathrm{Ph}$ & J.R.Lima, 39 & Stachyarrhena cf. spicata Hook. f. & Arv & $\mathrm{Ph}$ & J.R.Lima, 104 \\
\hline $\begin{array}{l}\text { Peltogyne confertiflora } \\
\text { (Mart. ex Hayne) Benth. }\end{array}$ & Arv & $\mathrm{Ph}$ & J.R.Lima, 50 & $\begin{array}{l}\text { Tocoyena formosa (Cham. \& } \\
\text { Schltdl.) K. Schum. }\end{array}$ & Arv & $\mathrm{Ph}$ & J.R.Lima, 59 \\
\hline Piptadenia moniliformis Benth. & Arv & $\mathrm{Ph}$ & F.S.Araújo, 1298 & RUBIACEAE A & Arv & $\mathrm{Ph}$ & J.R.Lima, 103 \\
\hline Senna cearensis Afr. Fern. & Arb & $\mathrm{Ph}$ & J.R.Lima, 46 & RUTACEAE & & & \\
\hline $\begin{array}{l}\text { S. trachypus (Mart. ex Benth.) } \\
\text { H.S. Irwin \& Barneby }\end{array}$ & Arb & $\mathrm{Ph}$ & J.R.Lima, 47 & $\begin{array}{l}\text { Galipea aff. trifoliata Aubl. } \\
\text { SALICACEAE }\end{array}$ & Arv & $\mathrm{Ph}$ & J.R.Lima, 36 \\
\hline $\begin{array}{l}\text { Swartzia flaemingii Raddi } \\
\text { LAMIACEAE }\end{array}$ & Arv & $\mathrm{Ph}$ & M.S.Sobrinho, 262 & $\begin{array}{l}\text { Xylosma ciliatifolia (Clos) Eichler } \\
\text { SAPINDACEAE }\end{array}$ & Arv & $\mathrm{Ph}$ & J.R.Lima, 77 \\
\hline $\begin{array}{l}\text { Vitex schaueriana Moldenke } \\
\text { MALPIGHIACEAE }\end{array}$ & Arv & $\mathrm{Ph}$ & J.R.Lima, 64 & $\begin{array}{l}\text { Paullinia cearensis Somner } \\
\quad \text { \& Ferrucci }\end{array}$ & Trp & $\mathrm{Ph}$ & J.R.Lima, 34 \\
\hline $\begin{array}{l}\text { Banisteriopsis stellaris (Griseb) } \\
\text { B. Gattes }\end{array}$ & $\operatorname{Trp}$ & $\mathrm{Ph}$ & J.R.Lima, 101 & $\begin{array}{l}P . \text { cf. elegans Cambess. } \\
\text { TRIGONIACEAE }\end{array}$ & Trp & $\mathrm{Ph}$ & J.R.Lima, 35 \\
\hline Byrsonima gardneriana A. Juss. & Arv & $\mathrm{Ph}$ & J.R.Lima, 32 & Trigonia nivea Cambess. & Trp & $\mathrm{Ph}$ & M.S.Sobrinho, 248 \\
\hline Peixotoa jussieuana Mart. ex & Trp & $\mathrm{Ph}$ & J.R.Lima, 33 & TURNERACEAE & & & \\
\hline A. Juss. & & & & Turnera blanchetiana Urb. & Arb & $\mathrm{Ph}$ & J.R.Lima, 65 \\
\hline MALVACEAE & & & & VERBENACEAE & & & \\
\hline Pavonia sp. & Erv & $\mathrm{Ch}$ & J.R.Lima, 90 & Lantana $\mathrm{cf}$. brasiliensis Link & Sub & $\mathrm{Ch}$ & J.R.Lima, 108 \\
\hline Helicteres heptandra L. B. Sm. & Arb & $\mathrm{Ph}$ & J.R.Lima, 51 & Lantana sp. & Sub & $\mathrm{Ch}$ & J.R.Lima, 109 \\
\hline
\end{tabular}

(7\%) e por criptófitos geófitos, hemicriptófitos e terófitos, que juntos, somaram $6 \%$ das espécies (Tab. 2). Não foi observada diferença significativa entre o espectro biológico da área estudada e da área de carrasco de Serras das Almas. O componente arbóreo e arbustivo foi representado por 76 espécies, distribuídas em 28 famílias. As trepadeiras foram 15 espécies, em nove famílias, sendo Bignoniaceae, a família com maior número de espécies (quatro espécies), seguida por Apocynaceae, Malpighiaceae e Sapindaceae (duas espécies cada). No componente subarbustivo ocorreram seis espécies, pertencentes a cinco famílias, sendo
Asteraceae exclusiva deste componente. As sete espécies herbáceas/sublenhosas pertenceram a cinco famílias (Tab. 1).

Similaridade florística - O dendrograma (Fig. 2) mostra a formação de três grupos significativos de acordo com o teste de simulação Monte Carlo, embora com baixa similaridade (limite superior $=0.16)$. O primeiro grupo $(\mathrm{A})$ uniu seis levantamentos sobre a Bacia Sedimentar do Meio Norte, além da área de floresta estudada, mostrando uma maior similaridade entre esta e as áreas de carrasco no Ceará (L2 a L5). O segundo grupo (B) foi formado por 
Tabela 2. Espectro biológico normal de Raunkiaer (1934) e da vegetação de áreas do semi-árido do Nordeste do Brasil. Formas de vida: Ch - caméfito; $\mathrm{H}$ - hemicriptófito; $\mathrm{Cg}$ - criptófito (geófito); $\mathrm{Ph}$ - fanerófito e Th - terófito. O valor crítico de $\chi^{2} 0,05 ; 4=9,49$. O valor do $\chi^{2}$ na comparação entre a área estudada/ espectro normal de Raunkiaer $=53,5 ;$ carrasco $=14,3$ e área estudada $=7,6$.

\begin{tabular}{|c|c|c|c|c|c|c|c|}
\hline \multirow[b]{2}{*}{ Área } & & \multicolumn{6}{|c|}{ Formas de Vida } \\
\hline & & $\mathrm{Ph}$ & $\mathrm{Ch}$ & $\mathrm{H}$ & $\mathrm{Cg}$ & $\mathrm{Th}$ & Total \\
\hline \multirow[t]{2}{*}{ Carrasco (Araújo et al. 2005b) } & $\mathrm{N}^{\circ}$ de espécies & 46 & 2 & 2 & 0 & 5 & 55 \\
\hline & $\%$ de espécies & 80,7 & 3,5 & 3,5 & 0 & 8,7 & 100 \\
\hline \multirow[t]{2}{*}{ Mata seca (este estudo) } & $\mathrm{N}^{o}$ de espécies & 91 & 7 & 3 & 1 & 2 & 104 \\
\hline & $\%$ de espécies & 87,5 & 6,7 & 2,8 & 1 & 2 & 100 \\
\hline Espectro Normal (Raunkiaer 1934) & $\%$ de espécies & 46 & 9 & 26 & 6 & 13 & 100 \\
\hline
\end{tabular}

duas áreas do Piauí, ambas também na Bacia do Meio Norte (L10, L11 e L16). O terceiro grupo (C) incluiu todos os levantamentos da Bacia Sedimentar Tucano-Jatobá, em Pernambuco, com exceção da área de Serra Negra (PE)

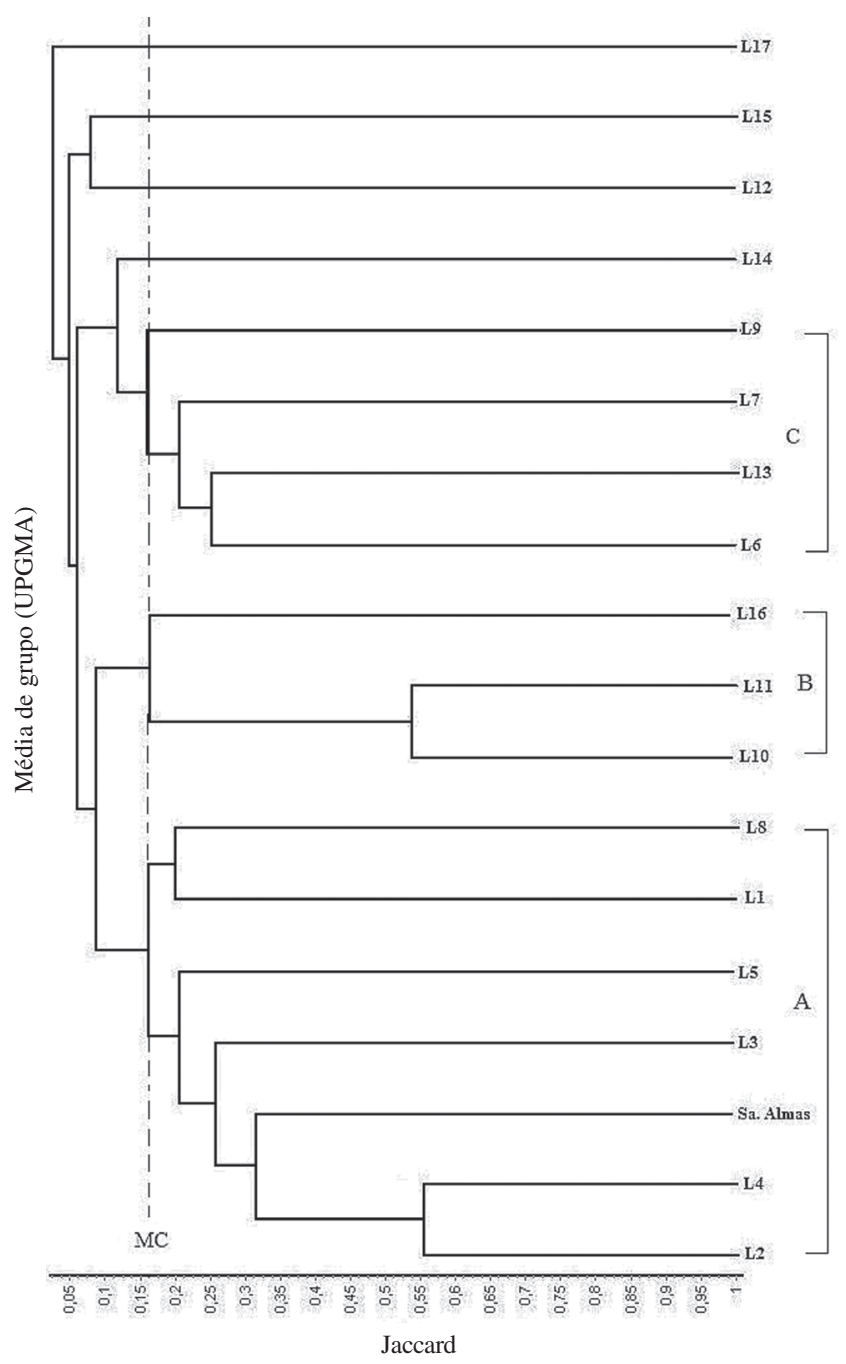

Figura 2. Similaridade florística entre a Floresta Estacional Decídua Montana da Serra das Almas e outras formações florísticas presentes em bacias sedimentares do semi-árido brasileiro. Os levantamentos (L) foram os seguintes: Oliveira et al. (1997): L1; Araújo et al. (1998a): L2 a L4; Araújo et al. (1999): L5; Rodal et al. (1999): L6; Figueirêdo et al. (2000): L7; Lemos \& Rodal (2002): L8; Andrade et al. (2004): L9; Farias \& Castro (2004): L10 e L11; Rocha et al. (2004): L12; Gomes et al. (2006): L13; Costa et al. (2004): L14; Maracajá et al. (2003): L15; M.R.A. Mendes (dados não publicados): L16 e Rodal \& Nascimento (2002): L17. (Correlação Cofenética = 1); MC: Monte Carlo.
(L17). Os levantamentos sobre a Chapada do Araripe (L14), Dunas de São Francisco (L12) e Apodi (L15), embora próximos no dendrograma, não formaram um grupo (similaridade inferior a 0.16). O teste de Mantel não mostrou correlação entre a distância geográfica e a similaridade florística $(r=-0,0386 ; g=-0,4804 ; \mathrm{p}=0,4120)$.

As áreas que apresentaram maior número de espécies em comum com a área estudada foram as localizadas na ecorregião Ibiapaba-Araripe (sensu Velloso et al. 2002), principalmente nas formações vegetais localizadas no planalto da Ibiapaba. Dentre os levantamentos encontrados nesta ecorregião, o que apresentou maior número de espécies em comum (56 espécies) com a área de estudo foi L5, uma área de carrasco.

\section{Discussão}

As famílias com maior número de espécies neste trabalho estão também entre as de maior riqueza nas florestas estacionais neotropicais, corroborando as observações de Gentry (1982; 1995). Estas famílias também são encontradas entre as de maior riqueza de espécies na caatinga (Araújo et al. 1995), exceto Myrtaceae e Bignoniaceae, bem como nas formações sobre as áreas sedimentares do Planalto da Ibiapaba. Embora estas famílias tenham ocorrido em todas as áreas, as espécies são diferentes, fato já destacado por Lemos \& Rodal (2002). Myrtaceae, além da alta representatividade nas formações sobre áreas sedimentares (ver Oliveira et al. 1997; Araújo et al. 1998b; Rodal et al. 1998; Araújo et al. 1999; Lemos \& Rodal 2002; Gomes et al. 2006), é uma família típica de matas úmidas serranas e costeiras do nordeste (Siqueira et al. 2001). Na caatinga, essa família tem sido registrada apenas nas áreas que apresentam maior umidade, já nos limites com outras formações (Pereira et al. 2002; Alcoforado Filho et al. 2003; Lourenço \& Barbosa 2003). Bignoniaceae está bem representada nas formações vegetais sobre a bacia sedimentar do Meio Norte estudadas por Oliveira et al. (1997), Araújo et al. (1998a; 1999) e Lemos \& Rodal (2002), mas tem baixa representatividade na caatinga, como ressaltado por Sampaio (1996).

A alta proporção de fanerófitos e a baixa proporção de terófitos na área estudada já eram esperadas, pois se trata de uma fisionomia florestal. A diferença não significativa entre o espectro biológico da área estudada e o do carrasco 
analisado (Araújo et al. 2005b) pode ser explicada pela alta proporção de espécies microfanerófiticas (46\%) encontradas na área de carrasco, que, juntamente com as espécies mesofanerófiticas, foram responsáveis por $75 \%$ das espécies da área, enquanto a floresta de Serra das Almas foi dominada por espécies mesofanerófiticas e megafanerófiticas (73\%). Embora esteja no domínio climático do semi-árido, a floresta estudada está localizada em área com maior disponibilidade hídrica. A precipitação corresponde ao limite máximo de delimitação do domínio semi-árido e está associada à maior altitude em relação às áreas de caatinga, resultando em menores temperaturas. A maior disponibilidade hídrica permite o estabelecimento de formas de vida que não necessitam de uma boa proteção das gemas de brotamento, estratégia necessária à sobrevivência em grande parte das espécies de regiões áridas e semi-áridas (ver Raunkiaer 1934; Van Rooyen et al. 1990; Kovács-Lang et al. 2000).

Retratando o encontrado no espectro biológico, a floresta de Serra das Almas teve um predomínio de plantas arbóreas e arbustivas (73\%), hábitos, conforme Medina (1995), dominantes em fisionomias florestais secas. Esse resultado também foi encontrado por Rodal \& Nascimento (2002), em duas áreas de floresta serranas do domínio semi-árido brasileiro. As trepadeiras lenhosas constituíram uma proporção considerável das espécies deste estudo (14\%) e foram distribuídas em 10 famílias, sendo consideradas por Gentry $(1982$; 1995) como um importante componente florístico nas florestas neotropicais, onde representam cerca de $20 \%$ das espécies.

A baixa riqueza do componente herbáceo, apenas sete espécies, é compatível com o esperado para fisionomias florestais, mesmo as decíduas. Diferentemente das florestas estacionais, a caatinga apresenta alta riqueza nesse componente, como observado por Barbosa et al. (2004) e Costa et al. (2007) que encontraram, respectivamente, $50 \mathrm{e}$ 77 espécies herbáceas, nas áreas que estudaram. Em regiões semi-áridas, tem sido observado um aumento na riqueza e abundância de espécies herbáceas e diminuição das espécies lenhosas à medida que a aridez aumenta (Pavón et al. 2000).

Os resultados da análise de agrupamento demonstraram a formação de três grupos estatisticamente significativos, constituídos principalmente por áreas com menor distância geográfica, independente do tipo de vegetação, embora com baixo nível de similaridade. O fato dos três grupos formados não serem explicados pela distância geográfica através do teste de Mantel possivelmente se deve à baixa similaridade dentro de cada grupo, o que reforça a hipótese de que há grande heterogeneidade espacial na flora de bacias sedimentares do semi-árido brasileiro. Assim, vegetações fisionomicamente diferentes, como a floresta estudada e o carrasco (vegetação arbustiva caducifólia não espinhosa), embora próximas, apresentaram baixa similaridade.
A maior semelhança da flora da floresta de Serra das Almas com as do carrasco localizado no topo do planalto da Ibiapaba parece indicar que é parte do mesmo domínio florístico. A flora de carrasco desceria a encosta leste até uma linha mais abaixo que a área estudada, onde faria uma transição mais ou menos abrupta com a flora de caatinga do cristalino da depressão. No entanto, as fisionomias da floresta e do carrasco são bem distintas. É possível, também, que as proporções dos indivíduos sejam diferentes, ainda que muitas das espécies presentes sejam as mesmas. Seria necessária uma avaliação fitossociológica das áreas para identificar se além da diferença no porte dos indivíduos, mais altos na floresta, também se traduz na importância relativa das espécies. Assim, a floresta estudada apresenta um conjunto florístico mais semelhante ao das formações encontradas sobre a Bacia Sedimentar do Meio Norte, mais especificamente do planalto da Ibiapaba, principalmente o carrasco.

\section{Agradecimentos}

À Coordenação de Aperfeiçoamento de Pessoal de Nível Superior (CAPES), pela bolsa concedida ao primeiro autor; ao Programa de Pós-Graduação em Botânica da Universidade Federal Rural de Pernambuco; ao Conselho Nacional de Pesquisa (CNPq), pelo financiamento do projeto (Edital Universal proc. 476285/2003-8); à Associação Caatinga pela infra-estrutura e apoio logístico nos trabalhos campo; aos biólogos Thiago da Ponte e Marcelo Oliveira Teles de Menezes, pela colaboração nas análises estatísticas dos dados e na confecção do mapa, respectivamente.

\section{Referências bibliográficas}

Alcoforado Filho, F.G.A.; Sampaio, E.V.S.B. \& Rodal, M.J.N. 2003. Florística e fitossociologia de um remanescente de vegetação caducifólia espinhosa arbórea em Caruaru, Pernambuco. Acta Botanica Brasilica 17: 287-303.

Andrade-Lima, D. 1981. The caatingas dominium. Revista Brasileira de Botânica 4: 149-153.

Andrade, K.V.S.A; Rodal, M.J.N.; Lucena, M.F.A. \& Gomes, A.P.S. 2004. Composição florística de um trecho do Parque Nacional do Catimbau, Buíque, Pernambuco - Brasil. Hoehnea 31: 337-348.

APG II. 2003. An update of the Angiosperm Phylogeny Group classification for the orders and families of flowering plants: APG II. Botanical Journal of the Linnaean Society 141: 399-436.

Araújo, E.L.; Sampaio, E.V.S.B. \& Rodal, M.J.N. 1995. Composição florística e fitossociológica de três áreas de caatinga. Revista Brasileira de Biologia 55: 595-607.

Araújo, F.S.; Sampaio, E.V.S.B.; Rodal, M.J.N. \& Figueiredo, M.A. 1998a. Organização comunitária do componente lenhoso de três áreas de carrasco em Novo Oriente, CE. Revista Brasileira de Biologia 58: 85-95.

Araújo, F.S.; Sampaio, E.V.S.B.; Figueiredo, M.A.; Rodal, M.J.N. \& Fernandes, A.G. 1998b. Composição florística da vegetação do carrasco, Novo Oriente, CE. Revista Brasileira de Botânica 21: $105-116$.

Araújo, F.S.; Martins, F.R. \& Shepherd, G.J. 1999. Variações estruturais e florísticas do carrasco no planalto da Ibiapaba, estado do Ceará. Revista Brasileira de Biologia 59: 663-678.

Araújo, F.S.; Rodal, M.J.N; Barbosa, M.R.V \& Martins, F.R. 2005a. Repartição da flora lenhosa no domínio da Caatinga. Pp. 15-33. In: F.S. Araújo, M.J.N. Rodal \& M.R.V. Barbosa (orgs). Análise das variações da biodiversidade do bioma caatinga: suporte a estratégias regionais de conservação. Brasília, Ministério do Meio Ambiente. 
Araújo, F.S.; Rodal, M.J.N.; Barbosa, M.R.V. \& Martins, F.R. 2005b. Vegetação e flora fanerogâmica da área Reserva Serra das Almas, Ceará. Pp. 91-120. In: F.S. Araújo, M.J.N. Rodal \& M.R.V. Barbosa (orgs). Análise das variações da biodiversidade do bioma caatinga: suporte a estratégias regionais de conservação. Brasília, Ministério do Meio Ambiente.

Barbosa, M.R.V.; Agra, F.M.; Sampaio, E.V.S.B.; Cunha, J.P. \& Andrade, L.A. 2004. Levantamento florístico preliminar do Pico do Jabre, Paraíba, Brasil. Pp. 111-122. In: K.C. Pôrto, J.J.P. Cabral \& M. Tabarelli (orgs.). Brejos de Altitude em Pernambuco e Paraíba: história natural, ecologia e conservação. Brasília, Ministério do Meio Ambiente.

Barroso, G.M.; Guimarães, E.F.; Ichaso, C.L.F.; Costa, C.G.; Peixoto, A.L. \& Lima, H.C. 1978. Sistemática de Angiospermas do Brasil 1. São Paulo, EDUSP.

Barroso, G.M.; Guimarães, E.F.; Ichaso, C.L.F.; Costa, C.G.; Peixoto, A.L. \& Lima, H.C. 1984. Sistemática de Angiospermas do Brasil 2. Viçosa, UFV.

Barroso, G.M.; Guimarães, E.F.; Ichaso, C.L.F.; Costa, C.G.; Peixoto, A.L. \& Lima, H.C. 1986. Sistemática de Angiospermas do Brasil 3. Viçosa, UFV.

Brasil, Projeto RADAMBRASIL. 1981. Mosaico semi-controlado de radar. Escala: 1: 250.000. Folhas SA-22-ZC, SB-22-XA, SB-22-XB e SB-22-SD. Rio de Janeiro, Departamento Nacional de Produção Mineral (DNPM).

Brummitt, R.K. \& Powell, C.E. 1992. Authors of plant names. Richmond, Kew Royal Botanic Gardens.

Cavalcante, A.M.B.; Soares, J.J. \& Figueiredo, M.A. 2000. Comparative phytosociology of tree sinusiae between contiguous forests in different stages of succession. Revista Brasileira de Biologia 60: 551-562.

Costa, I.R.; Araújo, F.S. \& Lima-Verde, L.W. 2004. Flora e aspectos autoecológicos de um encrave de cerrado na chapada do Araripe, Nordeste do Brasil. Acta Botanica Brasilica 18: 759-770.

Costa, R.C.; Araújo, F.S. \& Lima-Verde, L.W. 2007. Flora and life form spectrum in an area of a thorn deciduous woodland (caatinga) of Northeastern, Brazil. Journal of Arid Environments 68: 237-247.

Farias, R.R.S. \& Castro, A.A.J.F. 2004. Fitossociologia de trechos da vegetação do Complexo de Campo Maior, Campo Maior, PI, Brasil. Acta Botanica Brasilica 18: 949-963.

Ferraz, E.M.N. \& Rodal, M.J.N. 2006. Caracterização fisionômica estrutural de um remanescente de floresta ombrófila montana de Pernambuco, Brasil. Acta Botanica Brasilica 9: 11-926.

Ferraz, E.M.N. \& Rodal, M.J.N. 2008. Floristic characterization of a remnant ombrophilous montane forest at São Vicente Férrer, Pernambuco, Brazil. Memoirs of The New York Botanical Garden 100: 468-510.

Ferraz, E.M.N.; Rodal, M.J.N. \& Sampaio, E.V.S.B. 2003. Physiognomy and structure of vegetation along an altitudinal gradient in the semi-arid region of northeastern Brazil. Phytocoenologia 33: 71-92.

Ferraz, E.M.N.; Rodal, M.J.N.; Sampaio, E.V.S.B. \& Pereira, R.C.A. 1998. Variação florística ao longo de um gradiente altitudinal no alto vale do Pajeú, Pernambuco. Revista Brasileira de Botânica 21: 7-13.

Figueirêdo, L.S.; Rodal, M.J.N. \& Melo, A.L. 2000. Florística e fitossociologia de uma área de vegetação arbustiva caducifólia espinhosa no munícipio de Buíque - Pernambuco. Naturalia 25: 205-224.

Freire, C.V. 1983. Chaves analíticas para a determinação das famílias das plantas Pteridófitas, Gimnospermas e Angiospermas brasileiras ou exóticas cultivadas no Brasil. Volume 300. Mossoró, Coleção Mossoroense.

Gadelha Neto, P.C. \& Barbosa, M.R.V. 2000. Levantamento florístico e fitossociológico em um remanescente de caatinga no município de Sousa, Paraíba. Iniciados/UFPB 5: 64-87.
Gentry, A.H. 1982. Patterns of Neotropical plant species diversity. Evolutionary Biology 15: 1-84.

Gentry, A.H. 1995. Diversity and floristic composition of neotropical forest. Pp. 146-194. In: S.H Bullock, H.A. Mooney \& E. Medina (eds.) Seasonally dry tropical forest. Cambridge, Cambridge University Press.

Gomes, A.P.S; Rodal, M.J.N. \& Melo, A.L. 2006. Florística e fitossociologia da vegetação arbustiva subcaducifólia da Chapada de São José do Buique, Pernambuco, Brasil. Acta Botanica Brasilica 20: 1-12.

Kent, M. \& Coker, P. 1994. Vegetation description and analysis. A practical approach. Chichester, John Willey.

Kovács-Lang, E.; Kroel-Dulay, G.; Kertész, M., Fekete, G.; Bartha, S.; Mika, J.; Dobi-Wantuch, I.; Rédei, T.; Rajkai, K. \& Hahn, I. 2000. Changes in composition of sand grasslands along a gradient in Hungary and implications for climate change. Phytocoenologia 30: 385-407.

Krebs, C.J. 1989. Ecological methodology. New York, Harper \& Row.

Lemos, J.R. \& Rodal, M.J.N. 2002. Fitossociologia do componente lenhoso de um trecho da vegetação de caatinga no Parque Nacional da Serra da Capivara, Piauí, Brasil. Acta Botanica Brasilica 16: 23-42.

Liedloff, A. 1999. Mantel (Version 2.0): Mantel Nonparametric Test Calculator. Queensland, University of Technology.

Lins, R.C. 1978. A bacia do Parnaíba: aspectos fisiográficos. Recife, Instituto Joaquim Nabuco de Pesquisas Sociais.

Lourenço, L.E. \& Barbosa, M.R.V. 2003. Flora da fazenda Ipurana, Lagoa Seca, Paraíba (guia de campo). Revista Nordestina de Biologia 17: 23-58.

Maracajá, P.B.; Batista, C.H.F.; Sousa, A.H. \& Vasconcelos, W.E. 2003. Levantamento florístico e fitosociológico do extrato arbustivo- arbóreo de dois ambientes na Vila Santa Catarina, Serra do Mel, RN. Revista de Biologia e Ciências da Terra 3: 1-13.

Medina, E. 1995. Diversity of life forms of higher plants in neotropical dry forest. Pp. 221-238. In: S.H. Bullock, H.A. Mooney \& E. Medina (eds.). Seasonally dry tropical forest. Cambridge, Cambridge University Press.

Moura, F.B.P. \& Sampaio, E.V.S.B. 2001. Flora lenhosa de uma mata serrana semidecídua em Jataúba, Pernambuco. Revista Nordestina de Biologia 15: 77-89.

Nascimento, L.M.; \& Rodal, M.J.N. 2008. Fisionomia e estrutura de uma floresta estacional montana (brejo de altitude) do maciço do Borborema no nordeste do Brasil. Revista Brasileira de Botânica 31: 27-39.

Nascimento, C.E.S.; Rodal, M.J.N. \& Cavalcanti, A.C. 2003. Phytossociology of the remaining xerophytic woodland associated to an environmental gradient at the banks of the São Francisco river-Petrolina, Pernambuco, Brazil. Revista Brasileira de Botânica 26: 271.

Oliveira, M.E.A.; Sampaio, E.V.S.B.; Castro, A.A.J.F. \& Rodal, M.J.N. 1997. Flora e fitossociologia de uma área de transição carrascocaatinga de areia em Padre Marcos, Piauí. Naturalia 22: 131-150.

Pavón, N.P.; Hernández-Trejo, H. \& Rico-Gray, V. 2000. Distribution of life forms along an altitudinal gradient in the semi-arid valley of Zapotitlán, México. Journal of Vegetation Science 11: 22-39.

Pereira, I.M.; Andrade, L.A.; Barbosa, M.R.V \& Sampaio, E.V.S.B. 2002. Composição florística e análise fitossociológica do componente arbustivo-arbóreo de um remanescente florestal no agreste Paraibano. Acta Botanica Brasilica 16: 241-369.

Raunkiaer, C. 1934. The life forms of plants and statistical plant geography. Oxford, Clarendon Press. 
Rocha, P.L.B.; Queiroz, L.P. \& Pirani, R.J. 2004. Plant species and habitat structure in a sand dune field in the Brazilian caatinga: a homogeneous habitat harbouring an endemic biota. Revista Brasileira de Botânica 27: 739-755.

Rodal, M.J.N.; Andrade, K.V.A.; Sales, F.M. \& Gomes, A.P.S. 1998. Fitossociologia do componente lenhoso de um refúgio vegetacional no município de Buíque, Pernambuco. Revista Brasileira de Biologia 58: 517-526.

Rodal, M.J.N.; Nascimento, L.M. \& Melo, A.L. 1999. Composição florística de um trecho de vegetação arbustiva caducifólia, no município de Ibimirim, PE, Brasil. Acta Botanica Brasilica 13: $15-28$.

Rodal, M.J.N. \& Nascimento, L.M. 2002. Levantamento florístico da floresta serrana da Reserva Biológica de Serra Negra, microrregião de Itaparica, Pernambuco. Brasil. Acta Botanica Brasilica 16: 481-500.

Rodal, M.J.N.; Lins e Silva, A.C.B.; Pessoa, L.M. \& Cavalcanti, A.D.C. 2005. Vegetação e flora fanerogâmica da área de Betânia, Pernambuco. Pp. 91-119. In: F.S. Araújo, M.J.N. Rodal \& M.R.V. Barbosa (orgs). Análise das variações da biodiversidade do bioma caatinga: suporte a estratégias regionais de conservação. Brasília, Ministério do Meio Ambiente.

Sampaio, E.V.S.B. 1996. Fitossociologia. Pp. 203-230. In: E.V.S.B. Sampaio; J.S. Mayo \& M.R.V.Barbosa (eds.). Pesquisa Botânica Nordestina: Progresso e perspectivas. Recife, Sociedade Botânica do Brasil - Seção Regional de Pernambuco.

Shepherd, G.J. (2006). Fitopac V. Campinas, Universidade Estadual de Campinas - Departamento de Botânica.

Shimwell, D. W. 1971. The description and classification of vegetation. Seattle, University of Washington Press.
Siqueira, D.R.; Rodal, M.J.N.; Lins-e-Silva, A.C.B. \& Melo, A.L. 2001. Physiognomy, structure and floristic in an area of Atlantic Forest in Northeast Brazil. Pp.11-27. In: G. Gottsberger \& S. Liede (eds.). Life forms and dynamics in tropical forest. Berlin, J. Cramer.

Souza, M.J.N.; Martins, M.L.R.; Soares, Z.M.L.; Freitas-Filho, M.R.; Almeida, M.A.G.; Pinheiro, F.S.A.; Sampaio, M.A.B.; Carvalho, G.M.B.S.; Soares, A.M.L.; Gomes, E.C.B. \& Silva, R.A. 1994. Redimensionamento da região semi-árida do Nordeste do Brasil. Pp. 383-404. In: Conferência Nacional e Seminário Latino-Americano de Desertificação. Anais, Fortaleza. Brasília, Fundação Esquel do Brasil.

Tavares, M.C.G.; Rodal, M.J.N.; Lucena, M.F.A. \& Melo, A.L. 2000. Fitossociologia do componente arbóreo de um trecho de floresta ombrófila montana do parque ecológico João Vasconcelos Sobrinho, Caruaru, Pernambuco. Naturalia 25: 17-32.

Van Rooyen, M.W.; Theron, G.K. \& Grobbelaar, N. 1990. Life forms and spectra of flora of Namaqualand, South Africa. Journal of Arid Environments 19: 133-145.

Velloso, A.L.; Sampaio, E.V.S.B. \& Pareyn, F.G.C. 2002. Ecorregiões propostas para o Bioma caatinga. Recife, Associação Plantas do Nordeste, Instituto de Conservação Ambiental, The Nature Conservancy do Brasil.

Veloso, P.H.; Rangel Filho, A.L.R. \& Lima, J.C.A. 1991. Classificação da vegetação brasileira, adaptada a um sistema universal. Rio de Janeiro, IBGE.

Whittaker, R.H. 1975. Communities and ecosystems. New York, Macmillan. 